\title{
Eye Movement Distraction: A New Distraction Technique for Management of Dental Anxiety during Intraoral Local Anesthesia Administration in Children
}

\author{
Sunnypriyatham Tirupathi ${ }^{1}$, Nirmala Krishna ${ }^{2}$, Srinitya Rajasekhar ${ }^{3}$, Sivakumar Nuvvula ${ }^{4}$
}

\begin{abstract}
Background: To evaluate the efficacy of eye movement distraction (EMD) in reducing anxiety during intraoral local anesthetic administration in needle-phobic children.

Materials and methods: A total of 228 children aged 8-13 years were divided into two groups: group I (EMD) and group II (control), and subjective feelings of each child were recorded with a faces pain scale-revised (FPS-R) score. Both the groups (I and II) were compared using the Chi-square test.

Results: Children in the EMD group showed low FPS-R scores, which is statistically significant when compared to the control group ( $p<0.0001$ ). Conclusion: Eye movement distraction can be used as an effective distraction modality to reduce anxiety during local anesthesia administration in needle-phobic children.
\end{abstract}

Keywords: Anxiety control, Behavior management, Distraction, Local anesthesia.

International Journal of Clinical Pediatric Dentistry (2019): 10.5005/jp-journals-10005-1690

\section{INTRODUCTION}

Successful local anesthesia (LA) administration is important for many pediatric dental procedures. Administration of $L A$ itself is painful and anxiety-evoking, which can negatively influence the behavior of the child and may result in interruption of communication between the pediatric dentist and the child and avoidance of treatment. Hence, every effort should be made to reduce the pain and trauma during $L A$ administration. A number of chair side methods are used in daily practice to alleviate pain during LA administration such as topical anesthesia administration, slow rate of LA deposition, etc. Pain has both physiological and psychological components; psychological components of pain and anxiety should also be addressed. Distraction techniques address psychological components of pain by diverting the focus of the child from anxiety-evoking procedures. A variety of techniques are available, such as engaging the child in conversation, audio aids, audio-visual aids, deep breathing, music-guided imagery, WITAUL, and acupressure. ${ }^{1-7}$

\section{Aım}

To evaluate the efficacy of a new distraction technique, eye movement distraction (EMD), on pain behavior observed and reported by children receiving $L A$ injections prior to dental treatment.

\section{Materials and Methods}

A total of 228 children aged 8-13 years undergoing dental treatment at Department of Paedodontics, Narayana Dental College, Nellore, who required the administration of infiltration and who exhibited either a negative Frankl behavior rating scale or were needlephobic were recruited into the study after obtaining an informed consent and assent from parents and the children, respectively. Children were assigned randomly into two groups: group I (routine
${ }^{1}$ Department of Pedodontics and Preventive Dentistry, Malla Reddy Institute of Dental Sciences, Hyderabad, Telangana, India

${ }^{2}$ Department of Pedodontics, Narayana Dental College, Nellore, Andhra Pradesh, India

${ }^{3,4}$ Department of Paedodontics and Preventive Dentistry, Narayana Dental College and Hospital, Nellore, Andhra Pradesh, India

Corresponding Author: Sunnypriyatham Tirupathi, Department of Pedodontics and Preventive Dentistry, Malla Reddy Institute of Dental Sciences, Hyderabad, Telangana, India, Phone: +91-9490549454, e-mail: dr.priyatham@gmail.com

How to cite this article: Tirupathi S, Krishna N, Rajasekhar S, et al. Eye Movement Distraction: A New Distraction Technique for Management of Dental Anxiety during Intraoral Local Anesthesia Administration in Children. Int J Clin Pediatr Dent 2019;12(6):507-509.

Source of support: Nil

Conflict of interest: None

LA without EMD) and group II (LA + EMD). Topical anesthetic gel (Dologel) was applied to the injection site and left intact for 3 minutes.

Children in the EMD group were instructed on how to perform EMD for painless injection. The children were instructed before procedure that if at any point they stop performing EMD, then there are chances of increase in the pain. Children were asked to close their eyes and preform deep breathing followed by rotation of eyes in alternative clockwise and anti-clockwise directions. The children were instructed to count the number of rotations with their fingers. The children were kept actively distracted during administration of LA. Routine LA administration was done for children in the control group.

All injections were administered by a single operator. The LA solution was applied through 26 -gauge ( $38 \mathrm{~mm}$ in length) needles. 
Injection of the LA was slow, with an average duration of approximately $1 \mathrm{~mL} /$ minute. After conclusion of LA administration, the children were asked to complete the faces pain scale-revised (FPS-R) for subjective evaluation of feelings after the injection by a blinded examiner. The FPS-R included a set of six cartoon faces with varying facial expressions ranging from a smile/laughter to that of tears. Each face had a numerical value from 0 to 10 . The children selected the facial expression that best represented their experience of discomfort. Verbal instructions were given to the children on how to utilize the FPS-R. The behavior of the children was measured using the FPS-R. Faces pain scale-revised was compared in the study group and the control group.

Faces pain scale-revised score is a qualitative scale. In our study, we had further classified the subjective perception of child as category 1: more comfortable (FPS-R score $0-4$ ) and category 2: less comfortable (FPS-R score 6-10); further, both the groups (I and II) were compared using the Chi-square test with Yates correction. Significance was set at $p<0.05$. To reduce bias, age-matched controls were equally distributed between two groups.

\section{Results}

A total of 228 needle-phobic children (160 girls and 68 boys) were recruited for the study. To reduce bias, age matching was performed and equally distributed between two groups. Mean age of children was 10.5 years. Majority of children in both groups were above 10 years of age. Table 1 shows the FPS-R scoring of children of different ages in eye movement desensitization and reprocessing (EMDR) and control groups. Around $78.94 \%$ of children in the EMDR group had reported a subjective score of FPS-R (0-4). Around $21.05 \%$ of children in the EMDR group had reported a subjective score of FPS-R (6-10). Around $12.28 \%$ of children in the control group had reported a subjective score of FPS-R (0-4). Around $87.71 \%$ of children in the control group had reported a subjective score of FPS-R (6-10). A contingency table was prepared based on the available data. Results indicated that children in the EMDR group showed low FPS-R scores, which is statistically significant when compared to the control group $(p<0.0001)$ (Tables 2 and 3).

Table 1: Distribution of age and gender of children in various groups

\begin{tabular}{rcccc}
\hline Age & EMD-boys & $E M D$-girls & Control-boys & Control_girls \\
\hline 8 & 9 & 3 & 5 & 7 \\
9 & 0 & 0 & 0 & 0 \\
10 & 6 & 5 & 4 & 7 \\
11 & 7 & 27 & 9 & 25 \\
12 & 11 & 15 & 6 & 20 \\
13 & 9 & 22 & 2 & 29 \\
\hline
\end{tabular}

Table 2: The FPS-R scores of children in various groups

\begin{tabular}{lcrrcc}
\hline Subjective feeling & $F P S-R$ & $E M D-B$ & $E M D-G$ & Control-B & Control-G \\
\hline No hurt & 0 & 0 & 0 & 0 & 0 \\
Hurts little & 2 & 12 & 30 & 0 & 1 \\
Little more & 4 & 21 & 27 & 7 & 6 \\
Hurts more & 6 & 5 & 6 & 10 & 52 \\
Hurts severely & 8 & 2 & 7 & 9 & 22 \\
Worst pain & 10 & 2 & 2 & 0 & 7 \\
\hline
\end{tabular}

\section{Discussion}

This current study was planned to test the efficacy of EMD in reducing the anxiety of needle-phobic children who require administration of intraoral local anesthesia. The concept of EMD is derived from the Shapiros EMDR therapy, based on the theory that alternating bilateral stimulation or eye movement can reduce the fear and anxiety. Eye moment desensitization is one of the therapies used in psychological disorders such as post-traumatic stress disorders, panic disorders, and depression in both adults and children. Eye movement desensitization and reprocessing is an integrative therapy that "unlocks" disturbing memories or beliefs and reprocesses them, in some way, so they are no longer as disabling. Eye movement desensitization and reprocessing can be used for any experientially based psychological problems and has proven especially effective for traumatic imagery associated with post-traumatic stress disorder. ${ }^{8}$ Eye movement desensitization and reprocessing is a psychological therapy developed by Francine Shapiro. Francine Shapiro noticed that certain eye movements reduced the intensity of disturbing thoughts and stress. Shapiro noted that when she was experiencing a disturbing thought, her eyes were involuntarily moving rapidly; she noticed further that when she brought her eye movements under voluntary control while thinking a traumatic thought, anxiety was reduced. ${ }^{9}$ Khan et al. based on their systematic review reported that EMDR is better than cognitive behavioral therapy in patients with post-traumatic stress disorder. ${ }^{10}$ Lu et al. reported the use of alternating bilateral stimulation of eyes (a component of EMDR therapy) in fearful dental phobic patients who had a history of traumatic dental experience and reported that alternating bilateral stimulation (ABS) is effective in mild to moderate phobic patients. Alternating bilateral stimulation is simple and easy for patients and clinicians to perform during treatment and can be performed readily in the dental office. ${ }^{11}$ De Jongh et al. reported application of EMDR to traumatically induced dental phobia. Following two to three sessions of EMDR treatment, three of the four patients demonstrated substantially reduced self-reported and observerrated anxiety; EMDR can be an effective treatment alternative for phobic conditions with a trauma-related etiology. ${ }^{12}$

The eye movement component of EMDR therapy, also known as alternating bilateral stimulation, has been adopted in our study as it is simple and can be performed easily by children; reduction of anxiety is the main advantage of this therapy, although exact mechanism of it is not known clearly. ${ }^{13}$ Theory by MacCulloch and Feldman proposed that, inhibition of fear in eye movement component of EMDR therapy is due to activation of "investigatory reflex". This investigatory reflex has two stages, reflexive pause stage and reflexive exploration stage. Reflexive pause stage is the first stage and it produces strong sense of relaxation as well as it associates unpleasant memory

Table 3: Subjective opinion of children in various groups

\begin{tabular}{llc}
\hline & $E M D R$ & Control \\
\hline Comfortable & 90 & 14 \\
Painful & 24 & 100 \\
Chi-square static & 102.11 & \\
$p$ value & $<0.00001$ (highly significant) \\
\hline
\end{tabular}


with a pleasant visceral sensation. The second stage is reflexive exploratory stage where emotion and cognition is altered. ${ }^{14}$

Based on the results of our study, children in the EMD group reported less scores of FPS-R when compared to the control group and the difference was found to be statistically highly significant.

\section{Conclusion}

Eye movement distraction as a form of distraction can be used effectively in reducing the anxiety associated with intraoral needleprick pain in needle-phobic children aged 8-13 years.

Why it is important for pediatric dentists:

- The psychological component of dental anxiety can be addressed with this technique

- It does not require any additional equipment or materials

- It is not technique-sensitive and can be taught and performed chair side easily.

\section{ACKnOWLedgments}

We thank all the children for their participation in this study.

\section{References}

1. Kaur R, Jindal R, Dua R, et al. Comparative evaluation of the effectiveness of audio and audiovisual distraction aids in the management of anxious pediatric dental patients. J Indian Soc Pedod Prev Dent 2015;33(3):192-203. DOI: 10.4103/0970-4388.160357.

2. Nuvvula $S$, Alahari $S$, Kamatham $R$, et al. Effect of audiovisual distraction with $3 \mathrm{D}$ video glasses on dental anxiety of children experiencing administration of local analgesia: a randomised clinical trial. Eur Arch Paediatr Dent 2015;16(1):43-50. DOI: 10.1007/s40368014-0145-9.

3. Prabhakar AR, Marwah N, Raju OS. A comparison between audio and audiovisual distraction techniques in managing anxious pediatric dental patients. J Indian Soc Pedod Prev Dent 2007;25(4):177-182. DOI: 10.4103/0970-4388.37014.
4. Peretz B, Gluck GM. Assessing an active distracting technique for local anesthetic injection in pediatric dental patients: repeated deep breathing and blowing out air. J Clin Pediatr Dent 1999;24(1):5-8.

5. Bradt J, Teague A. Music interventions for dental anxiety. Oral Dis 2018;24(3):300-306. DOI: 10.1111/odi.12615.

6. Kamath PS. A novel distraction technique for pain management during local anesthesia administration in pediatric patients. J Clin Pediatr Dent 2013;38(1):45-47. DOI: 10.17796/jcpd.38.1.265807t236 570 hx7.

7. Avisa $P$, Kamatham R, Vanjari K, et al. Effectiveness of acupressure on dental anxiety in children. Pediatr Dent 2018;40(3):177-183.

8. Lee GK, Beaton RD, Ensign J. Eye movement desensitization and reprocessing. A brief and effective treatment for stress. J Psychosoc Nurs Ment Health Serv 2003;41(6):22-31.

9. Shapiro F. The role of eye movement desensitization and reprocessing (EMDR) therapy in medicine: addressing the psychological and physical symptoms stemming from adverse life experiences. Perm J 2014;18(1):71-77. DOI: 10.7812/TPP/13-098.

10. Khan AM, Dar S, Ahmed R, et al. Cognitive behavioral therapy versus eye movement desensitization and reprocessing in patients with post-traumatic stress disorder: systematic review and meta-analysis of randomized clinical trials. Cureus 2018;10(9):e3250. DOI: 10.7759/ cureus. 3250 .

11. Lu DP. Using alternating bilateral stimulation of eye movement desensitization for treatment of fearful patients. Gen Dent 2010;58(3):e140-e147.

12. De Jongh $A$, van den Oord $\mathrm{HJ}$, ten Broeke E. Efficacy of eye movement desensitization and reprocessing in the treatment of specific phobias: four single-case studies on dental phobia. J Clin Psychol 2002;58(12):1489-1503. DOI: 10.1002/jclp.10100.

13. Landin-Romero R, Moreno-Alcazar A, Pagani M, et al. How does eye movement desensitization and reprocessing therapy work. A systematic review on suggested mechanisms of action. Front Psychol 2018;9:1395. DOI: 10.3389/fpsyg.2018.01395.

14. MacCulloch MJ, Feldman P. Eye movement desensitisation treatment utilises the positive visceral element of the investigatory reflex to inhibit the memories of post-traumatic stress disorder: a theoretical analysis. Br J Psychiatry 1996;169(5):571-579. DOI: 10.1192/ bjp.169.5.571. 